\title{
Influence of 4-CPA Growth Regulator for Enhancing Yield of Tomato During Low Night Temperature Stress
}

\author{
Saeed Ahmed Shah Chishti ${ }^{1}$, Saba Aleem ${ }^{*}$, Iram Sharif ${ }^{2}$, Kashif Nadeem ${ }^{1}$, Nusrat Parveen ${ }^{1}$ and \\ Muhammad Najeebullah ${ }^{1}$
}

${ }^{1}$ Vegetable Research Institute, Ayub Agricultural Research Institute, Faisalabad, Pakistan; ${ }^{2}$ Cotton Research Station, Ayub Agricultural Research Institute, Faisalabad, Pakistan.

\begin{abstract}
Flowering in Solanum lycopersicum L. is sensitive to low-temperature as it inhibits the fuit growth and causes poor fruit setting. The present experiment was conducted to improve the fruit growth and fruit set in tomato during low-temperature stress conditions by the use of growth regulator 4-chlorophenoxy acetic acid. Seven different 4-chlorophenoxy acetic acid levels along with two indeterminate tomato varieties saandal and sahel were tested in two minimum night temperature conditions $12.5-23^{\circ} \mathrm{C}$ and $2-7.5^{\circ} \mathrm{C}$. Tomato yield per plant, yield per cluster, number of fruits per cluster, fruit weight and fruit diameter were significantly improved by the application of 4-chlorophenoxy acetic acid. Highest yield per plant $13.50 \mathrm{~kg}$ was recorded with the application of $75 \mathrm{ppm}$ in 4- chlorophenoxy acetic acid twicely when the minimum temperature was ranged between $12.5-23^{\circ} \mathrm{C}$. Likewise, maximum yield per plant $7.08 \mathrm{~kg}$ was also obtained with the same treatment when the minimum temperature was between $2-7.5^{\circ} \mathrm{C}$. This gain was primarily due to increased yield per cluster, fruits per cluster, fruit weight and fruit diameter in both minimum night temperature conditions $\left(12.5-23^{\circ} \mathrm{C}\right.$ and $\left.2-7.5^{\circ} \mathrm{C}\right) .87 \%$ gain in yield per plant was observed with $75 \mathrm{ppm}$ double sprayed treatment over control when minimum night temperature varied between $2-7.5^{\circ} \mathrm{C}$. Successful yield increase per plant suggested that 4-CPA could be used as a tool for getting economical tomato yield when night temperature falls below $10^{\circ} \mathrm{C}$.

Received | April 21, 2019; Accepted | March 21, 2020; Published | April 20, 2020

*Correspondence | Saba Aleem, Vegetable Research Institute, Ayub Agricultural Research Institute, Faisalabad, Pakistan; Email: sabaaleem22@ gmail.com

Citation | Chishti, S.A.S., S. Aleem, I. Sharif, K. Nadeem, N. Parveen and M. Najeebullah. 2020. Influence of 4-CPA growth regulator for enhancing yield of tomato during low night temperature stress. Pakistan Journal of Agricultural Research, 33(2): 217-223.

DOI | http://dx.doi.org/10.17582/journal.pjar/2020/33.2.217.223

Keywords | 4-Chlorophenoxy acetic acid, Low night temperature, Parthenocarpy, Tomato, Yield
\end{abstract}

\section{Introduction}

$\mathrm{A}^{\mathrm{t}}$ the flowering stage tomato (Solanum Alycopersicum L.) is very prone to both high and low temperatures. Studies conducted to find the relationship between daily temperature and reproductive stage of tomato have found that day temperatures of $21-28^{\circ} \mathrm{C}$ and night temperatures of $15-20{ }^{\circ} \mathrm{C}$ are optimal for proper fruit setting and size development in tomato (Rahman et al., 2015).
Any increase or decrease from optimum temperature results in premature drop of tomato fruit (Pramanik et al., 2017). Night temperature also plays an important role in the setting and development of the fruit, as night temperature below $10{ }^{\circ} \mathrm{C}$ significantly affects the fruit set in tomato (Karapanos et al., 2008). Adam et al. (2001) while evaluating the impact of temperature on growth and development of tomato fruit, reported that temperature below $14^{\circ} \mathrm{C}$ greatly reduces the tomato growth and causes the June 2020 | Volume 33 | Issue 2 | Page 217 
development of small and parthenocarpic tomatoes. Low temperature mainly reduces the pollen viability, pollen release and also delays germination of pollen on stigma, pollen tube development in style that leads to poor pollination and delays the fertilization process which are the prerequisite for proper fruit setting and development in tomato (Fernandez-munoz et al., 1995; Pressman et al., 2007).

Plant growth regulators are extensively being practiced in tomato for improving crop yield in temperature stress conditions. The impact of synthetic auxins and gibberellins to boost the production of tomatoes by setting parthenocarpic fruits has been acknowledged by many researchers (Tiwari et al., 2014; Pramanik et al., 2017). 4-Chlorophenoxy acetic acid is a synthetic auxin whose application has been reported to increase fruit set and size of tomato in temperature stress conditions (Picken and Grimmett, 1986; Ramin, 2003).

Many studies tested the growth hormones applications to improve tomato crop yield during high-temperature stress. There are few studies that check its effectiveness in low-temperature stress. However, investigations regarding 4-CPA test for low night temperature conditions are limited. In the present study effects of different 4-CPA levels were assessed in response to two minimum temperature conditions. In the first condition, different 4-CPA levels were tested during October to November when minimum night temperature was ranging between $\left(12.5^{\circ} \mathrm{C}-23^{\circ} \mathrm{C}\right)$ and secondly the same levels of 4-CPA were tested in December- January when the minimum temperature at night was ranging between $\left(2^{\circ} \mathrm{C}-7.5^{\circ} \mathrm{C}\right)$. The ultimate goal of the study was to find the best suitable level of 4-CPA growth regulator that could improve the fruit set and yield of the tomato crop during minimum nigh temperature conditions.

\section{Materials and Methods}

The experiment was conducted at the research area of Vegetable Research Institute Faisalabad, Pakistan, located at $31^{\circ} 23{ }^{\prime} 52.8^{\prime \prime} \mathrm{N}$ and $73^{\circ} 02$ '56.9” E during August 2017 to April 2018. Two indeterminate tomato hybrids Saandal and Sahel were used in this study. Both these varieties are being used commercially in normal planting season. Sandals give early production, but Sahel's fruit size is larger than sandals. During the last week of August 2017, seeds of both varieties were sown in vermicompost filled trays and seedlings of each variety were transplanted on both sides of the two beds in the last week of September in the high tunnel with the dimension of $(100 \mathrm{ft} \times 30 \mathrm{ft} \times 15 \mathrm{ft})$. Two-factor randomized complete block design with five replications was used to design the experiment and a flower cluster with 3 opened flowers was treated as an experimental unit. All the recommended cultural practices were followed during the conduction of this experiment.

The spray of 4-CPA treatments was performed five times with a one-week interval from October to November (18th Oct- 15th Nov) when minimum night temperature was ranging between $\left(12.5^{\circ} \mathrm{C}-23^{\circ} \mathrm{C}\right)$. Secondly, the same experiment was repeated in December-January (28th Dec to 26th Jan) when the minimum night temperature was ranging between $\left(2^{\circ} \mathrm{C}-7.5^{\circ} \mathrm{C}\right)$. Seven 4 - chlorophenoxy acetic acid (4-CPA) levels i.e. T1 $=00$ ppm (control), T2 $=25 \mathrm{ppm}$ single spray, T3= $50 \mathrm{ppm}$ single spray, $\mathrm{T} 4=75 \mathrm{ppm}$ single spray, $\mathrm{T} 5=25 \mathrm{ppm}$ double spray, $\mathrm{T} 6=50 \mathrm{ppm}$ double spray, $\mathrm{T} 7=75 \mathrm{ppm}$ double spray were used to access the effect of different levels of 4-CPA. In single spray treatments selected clusters were sprayed only one time while in double spray treatments, clusters were sprayed twice.

To collect data regarding tomato yield, all ripened fruits of each treatment were harvested separately. To collect data for fruit per cluster, all the marketable size ripened fruits were counted. On average five fruits from the center of the cluster of each treatment were used for data collection for fruit weight, fruit diameter, and the number of seed per fruit.

Analyses of variance for all the characters were performed using Statistix software. Means of all the treatments were calculated and the significance of differences between the pairs of means was tested by using the least significant difference test at $5 \%$ level of significance. Percentage increase and decrease by the use of dose (double spray $75 \mathrm{ppm}$ ) that give more increase in traits over control was also calculated to get a more clear picture of the effect of 4-CPA.

\section{Results and Discussion}

\section{Yield per plant}

Yield per plant is a complex trait and is highly correlated with other traits such as fruit weight, fruit diameter 
and the number of fruit set per cluster. In the present study, yield per plant was significantly improved when minimum night temperature was between $2^{\circ} \mathrm{C}-7.5^{\circ} \mathrm{C}$ by the application of growth regulator 4-CPA. A comparison of means showed significant differences in response to the different levels of 4-CPA. During cooly nights when the temperature was between $2^{\circ} \mathrm{C}$ $-7.5^{\circ} \mathrm{C}$, highest yield per plant $(7.08 \mathrm{~kg})$ was recorded by the use of $75 \mathrm{ppm}$ dose twice in a week (Table 3). $87 \%$ more yield was recorded in double sprayed 75 ppm treatment than control (Table 1). In single spray treatment, $75 \mathrm{ppm}$ produced the highest yield (5.91 $\mathrm{kg})$ as compared to other treatments i.e $25 \mathrm{ppm}(4.18$ $\mathrm{kg}$ ) and $50 \mathrm{ppm}(4.35 \mathrm{~kg})$. Control treatment (0 ppm) showed the minimum yield $(3.78 \mathrm{~kg})$ as compared to other treatments (Table 3 ). Both varieties also showed significant differences regarding yield per plant. Saandal variety showed more yield per plant $(4.74 \mathrm{~kg})$ than the Sahel $(4.30 \mathrm{~kg})$ when night temperature was $2^{\circ} \mathrm{C}-7.5^{\circ} \mathrm{C}$.

When the temperature was between $12.5-23^{\circ} \mathrm{C}$, then again the application of growth regulator was found effective. Yield per plant was increased by the use of growth regulator 4-CPA in this condition. The interaction effects of variety into 4-CPA levels were found non-significant and indicated that the main effects of variety and 4-CPA levels are more important. Differences in varieties in respect of yield per plant were non-significant when minimum night temperature was $12.5-23^{\circ} \mathrm{C}$. Significant differences in means of different 4-CPA levels indicated that to improve the yield of tomato use of 4-CPA is effective when night temperature was between $12.5-23^{\circ} \mathrm{C}$. Highest yield per plant was recorded with $75 \mathrm{ppm}$ double spray treatment and minimum was found in control. In single spray treatment, $75 \mathrm{ppm}(10.51 \mathrm{~kg})$ was found more effective than other treatments i.e. 25 ppm $(7.53 \mathrm{~kg})$, and $50 \mathrm{ppm}(8.22 \mathrm{~kg})$. Double spray treatment of $25 \mathrm{ppm}(8.46 \mathrm{~kg})$ and $50 \mathrm{ppm}(9.69 \mathrm{~kg})$ showed more yield than single spray treatment $25 \mathrm{ppm}$ $(7.53 \mathrm{~kg})$ and $50 \mathrm{ppm}(8.22 \mathrm{~kg})$ (Table 3). Increase yield per plant was mainly due to the increase in fruit set and fruit growth of tomato. Picken and grimmett, 1986 found a small increase in yield by the application of 4-CPA at the rate of $0.02 \mathrm{gl}^{-1}$ and $\beta$ napthoxy acetic acid $0.0075 \mathrm{gl}^{-1}$ in tomato. They found that $\beta$ napthoxy acetic acid is effective at $16^{\circ} \mathrm{C}$ and $4-\mathrm{CPA}$ at $13^{\circ} \mathrm{C}$ minimum night temperature. Further, the effect of both these growth regulators was questionable when minimum night temperature was $11^{\circ} \mathrm{C}$. Our results varied from their findings as we recorded an increase of $87 \%$ in yield when minimum night temperature was $2^{\circ} \mathrm{C}-7.5^{\circ} \mathrm{C}$. This increase in yield was may be due to the use of higher concentration $75 \mathrm{ppm}\left(0.075 \mathrm{gl}^{-1}\right)$ and twice spray in a week.

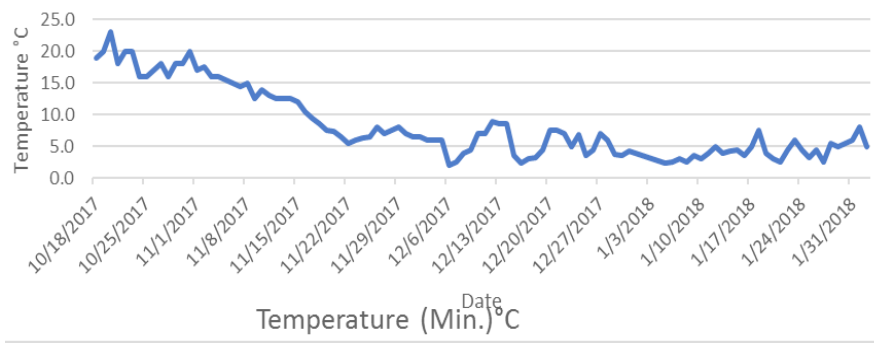

Figure 1: Minimum night temperature recorded during the spraying period from Mid-October to January.

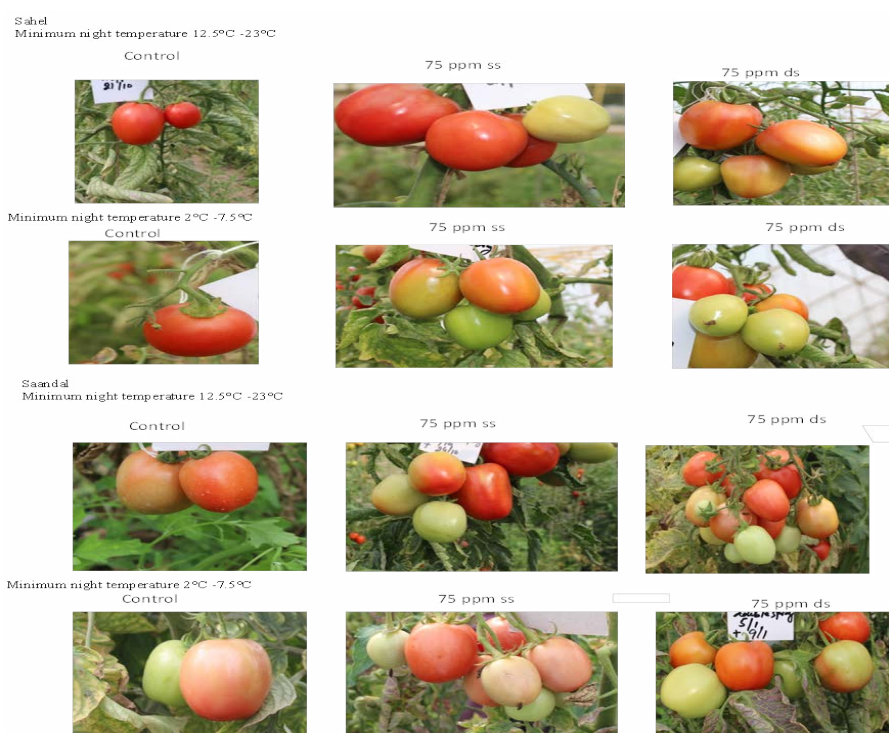

Figure 2: Impact of $75 \mathrm{ppm}$ single as well as double spray over control in both temperature conditions.

\section{Tomato yield per cluster}

Tomato yield in both temperature conditions was significantly increased by the application of plant growth regulator 4-CPA. This increase in yield was mainly due to an increase in the number of fruits set on a cluster, fruit weight, and fruit diameter. $75 \mathrm{ppm}$ double spray treatment in both temperature conditions $12.5^{\circ} \mathrm{C}-23^{\circ} \mathrm{C}$ and $2^{\circ} \mathrm{C}-7.5^{\circ} \mathrm{C}$ produced more yield per cluster as compared to control (0 ppm). An overall 163-300 \% increase in yield was recorded in sprayed clusters over control in both temperature conditions $\left(12.5^{\circ} \mathrm{C}-23^{\circ} \mathrm{C}\right.$ and $2^{\circ} \mathrm{C}-7.5^{\circ} \mathrm{C}$ ) (Table 1). The highest yield of $1.12 \mathrm{~kg}$ and $0.58 \mathrm{~kg}$ per cluster was recorded respectively, for both minimum night temperature conditions $\left(12.5^{\circ} \mathrm{C}-23^{\circ} \mathrm{C}\right.$ and $\left.2^{\circ} \mathrm{C}-7.5^{\circ} \mathrm{C}\right)$ when the genotypes were sprayed with $75 \mathrm{ppm}$ twice while lowest yield was observed in control $(0.42 \mathrm{~kg}, 0.14$ $\mathrm{kg}$ ) as presented in Table 3. In single spray treatment, 
Table 1: Percentage increase/decrease in $75 \mathrm{ppm}$ double sprayed treatmetnt over control in respect of different studied parameters.

\begin{tabular}{|c|c|c|c|c|c|c|}
\hline \multirow[t]{2}{*}{ Character } & \multicolumn{3}{|c|}{ Min. temperature ranging between $12.5^{\circ} \mathrm{C}-23^{\circ} \mathrm{C}$} & \multicolumn{3}{|c|}{ Min. temperature ranging between $2^{\circ} \mathrm{C}-7.5^{\circ} \mathrm{C}$} \\
\hline & Control & Sprayed & $\begin{array}{l}\text { Percentage increase/ } \\
\text { Decrease }\end{array}$ & $\begin{array}{l}\text { Control } \\
\text { treatment }\end{array}$ & $\begin{array}{l}\text { Sprayed } \\
\text { treatment }\end{array}$ & $\begin{array}{l}\text { Percentage increase/ } \\
\text { Decrease }\end{array}$ \\
\hline Yield per plant & 6.49 & 12.00 & 85 & 3.78 & 7.08 & 87 \\
\hline Yield per cluster $(\mathrm{kg})$ & 0.428 & 1.124 & 163 & 0.147 & 0.589 & 300 \\
\hline Number of fruits per cluster & 3.70 & 6.80 & 83 & 2.50 & 6.00 & 140 \\
\hline Fruit weight (g) & 113.29 & 167.84 & 48 & 58.30 & 96.17 & 65 \\
\hline Fruit Diameter (mm) & 52.38 & 67.46 & 29 & 45.71 & 61.29 & 34 \\
\hline No. of seeds/ fruit & 57.05 & 20.70 & 63 & 49.10 & 9.50 & 8 \\
\hline
\end{tabular}

Table 2: Performance of different tomato varieties in low night temperature stress.

\begin{tabular}{|c|c|c|c|c|c|c|c|c|c|c|c|c|}
\hline \multirow[t]{2}{*}{ Treatments } & \multicolumn{6}{|c|}{$\begin{array}{l}\text { Minimum night temperature ranging between } \\
12.5^{\circ} \mathrm{C}-23^{\circ} \mathrm{C}\end{array}$} & \multicolumn{6}{|c|}{$\begin{array}{l}\text { Minimum night temperature ranging between } \\
2^{\circ} \mathrm{C}-7.5^{\circ} \mathrm{C}\end{array}$} \\
\hline & $\mathrm{NF} / \mathrm{C}$ & FW (g) & $\begin{array}{l}\text { FD } \\
(\mathrm{mm})\end{array}$ & $\mathrm{NS} / \mathrm{F}$ & $\begin{array}{l}\mathrm{Y} / \mathrm{C} \\
(\mathrm{kg})\end{array}$ & $\begin{array}{l}\mathrm{Y} / \mathrm{P} \\
(\mathrm{kg})\end{array}$ & $\mathrm{NF} / \mathrm{C}$ & $\begin{array}{l}\text { FW } \\
(\mathrm{g})\end{array}$ & $\begin{array}{l}\text { FD } \\
(\mathrm{mm})\end{array}$ & $\mathrm{NS} / \mathrm{F}$ & $\begin{array}{l}\mathrm{Y} / \mathrm{C} \\
(\mathrm{kg})\end{array}$ & $\begin{array}{l}\mathrm{Y} / \mathrm{P} \\
(\mathrm{kg})\end{array}$ \\
\hline V1 (Sahel) & $4.68 b$ & $151.89 \mathrm{a}$ & $64.06 \mathrm{a}$ & $33.72 \mathrm{a}$ & $0.72 \mathrm{a}$ & $8.72 \mathrm{a}$ & $4.34 \mathrm{~b}$ & $77.27 \mathrm{~b}$ & $51.82 \mathrm{~b}$ & $19.25 b$ & $0.35 \mathrm{~b}$ & $4.30 \mathrm{~b}$ \\
\hline V2 (Saandal) & $5.74 \mathrm{a}$ & $129.80 \mathrm{~b}$ & $60.24 b$ & $33.45 \mathrm{a}$ & $0.75 \mathrm{a}$ & $9.02 \mathrm{a}$ & $4.85 \mathrm{a}$ & $79.27 \mathrm{a}$ & $55.05 \mathrm{a}$ & $27.74 \mathrm{a}$ & $0.39 a$ & $4.74 \mathrm{a}$ \\
\hline LSD & 0.21 & 4.08 & 1.11 & 1.72 & 0.04 & 0.50 & 0.18 & 1.22 & 0.81 & 1.34 & 0.01 & 0.15 \\
\hline Level of significance (5\%) & * & * & * & ns & ns & ns & * & * & * & * & * & * \\
\hline
\end{tabular}

NF/C: Number of fruit per cluster; FW: Fruit weight; FD: Fruit Diameter; NS/F: No. of seed/ fruit; Y/C: Yield per cluster; Y/P: yield per plant.

Table 3: Impact of different 4-CPA levels on different yield contributing characters of tomato during low night temperature.

Treatments

\begin{tabular}{|c|c|c|c|c|c|c|c|c|c|c|c|c|}
\hline & 6.5 & & & & & & & & & & & \\
\hline & $\mathrm{JF} / \mathrm{C}$ & $\begin{array}{l}\text { FW } \\
(\mathrm{g})\end{array}$ & $\begin{array}{l}\text { FD } \\
(\mathrm{mm})\end{array}$ & NS/F & $\begin{array}{l}\mathrm{Y} / \mathrm{C} \\
(\mathrm{kg})\end{array}$ & $\begin{array}{l}\mathrm{Y} / \mathrm{P} \\
(\mathrm{Kg})\end{array}$ & $\mathrm{NF} / \mathrm{C}$ & $\begin{array}{l}\text { FW } \\
(\mathrm{g})\end{array}$ & FD & $\mathrm{NS} / \mathrm{F}$ & $\begin{array}{l}\text { Y/C } \\
(\mathrm{kg})\end{array}$ & $\begin{array}{l}\mathrm{Y} / \mathrm{P} \\
(\mathrm{kg})\end{array}$ \\
\hline (0ppm) & $3.70 f$ & $113.29 \mathrm{e}$ & $52.38 \mathrm{f}$ & 5 & $0.42 \mathrm{e}$ & $6.49 \mathrm{e}$ & e & $58.30 \mathrm{~g}$ & & $0 \mathrm{a}$ & $0.14 f$ & $3.78 \mathrm{f}$ \\
\hline $2(25 \mathrm{ppm}$ & $4.50 \mathrm{e}$ & $125.40 \mathrm{~d}$ & $59.20 \mathrm{e}$ & $43.10 \mathrm{~b}$ & $0.54 \mathrm{~d}$ & $7.53 \mathrm{~d}$ & $3.80 \mathrm{~d}$ & $67.61 \mathrm{f}$ & $50.24 \mathrm{e}$ & $28.00 \mathrm{~b}$ & $0.26 \mathrm{e}$ & $3.98 \mathrm{e}$ \\
\hline $3(5$ & $5.30 \mathrm{c}$ & $132.34 d$ & $9 d$ & $33.60 \mathrm{c}$ & $0.68 c$ & $8.22 \mathrm{c}$ & $4.70 \mathrm{c}$ & $77.74 d$ & $\mathrm{~cd}$ & $22.40 \mathrm{~d}$ & $0.36 \mathrm{~d}$ & $4.35 \mathrm{~d}$ \\
\hline 4 (75 ppm sir & $5.90 \mathrm{~b}$ & $147.02 \mathrm{c}$ & $65.25 \mathrm{~b}$ & $26.20 \mathrm{~d}$ & $0.87 \mathrm{~b}$ & $10.51 \mathrm{~b}$ & $5.40 \mathrm{~b}$ & $89.97 \mathrm{~b}$ & & $16.20 \mathrm{f}$ & $0.49 \mathrm{~b}$ & $5.91 \mathrm{~b}$ \\
\hline $5(25 \mathrm{ppm} \mathrm{c}$ & $4.90 \mathrm{~d}$ & $145.26 \mathrm{c}$ & & & & $8.46 \mathrm{c}$ & $\mathrm{c}$ & $74.71 \mathrm{e}$ & & & $0.33 \mathrm{~d}$ & 4.08 \\
\hline$(50 \mathrm{ppm} \mathrm{c}$ & $.40 \mathrm{c}$ & $154.77 \mathrm{~b}$ & $65.25 \mathrm{~b}$ & $25.80 \mathrm{~d}$ & $0.80 \mathrm{~b}$ & $9.69 \mathrm{~b}$ & $5.30 \mathrm{~b}$ & $83.30 \mathrm{c}$ & & & $0.44 \mathrm{c}$ & $.31 c$ \\
\hline$(75 \mathrm{ppm} \mathrm{c}$ & $6.80 \mathrm{a}$ & $167.84 \mathrm{a}$ & $67.46 \mathrm{a}$ & $20.70 \mathrm{e}$ & $1.12 \mathrm{a}$ & $12.00 \mathrm{a}$ & $6.00 \mathrm{a}$ & $96.17 \mathrm{a}$ & $9 \mathrm{a}$ & $9.50 \mathrm{~g}$ & $.58 \mathrm{a}$ & $7.08 \mathrm{a}$ \\
\hline $\mathrm{D}$ & & 7.6 & 0. & 3. & 0. & 0 . & 0 & 2.2 & 1. & 2. & 0.02 & 0.29 \\
\hline vel of significance (5\%) & * & $*$ & $*$ & $*$ & $*$ & 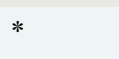 & $*$ & 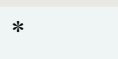 & $*$ & $*$ & 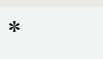 & * \\
\hline
\end{tabular}

NF/C: Number of fruit per cluster; FW: Fruit weight; FD: Fruit Diameter; NS/F: No. of seed fruit; Y/C: Yield per cluster; Y/P: yield per plant.

$75 \mathrm{ppm}$ produced the highest yield $(0.49 \mathrm{~kg})$ as compared to other treatments i.e. $0 \mathrm{ppm}(0.14 \mathrm{~kg})$, $25 \mathrm{ppm}(0.26 \mathrm{~kg})$, and $50 \mathrm{ppm}(0.36 \mathrm{~kg})$ when crop faced the temperature of $2^{\circ} \mathrm{C}-7.5^{\circ} \mathrm{C}$ at night time (Table 3). Control treatment $(0 \mathrm{ppm})$ showed the minimum yield per cluster $(0.14 \mathrm{~kg})$ as compared to other treatments. Double sprayed treatments also showed more yield per cluster than single spray when minimum night temperature was between $2^{\circ} \mathrm{C}-7.5^{\circ} \mathrm{C}$. Significant differences were also observed between varieties regarding yield per cluster. Saandal hybrid $(0.35 \mathrm{~kg})$ gave more yield per cluster than Sahel $(0.35 \mathrm{~kg})$. The performance of the varieties under different treatments showed that the variety Saandal is more responsive to the different levels of 4-CPA than Sahel (Table 4). 
Table 4: Comparison of interaction effects of 4-CPA levels and varieties in respect of different yield contributing characters of tomato during low night temperature stress.

\begin{tabular}{|c|c|c|c|c|c|c|c|c|c|c|c|c|}
\hline \multirow[t]{2}{*}{ Treatments } & \multicolumn{6}{|c|}{$\begin{array}{l}\text { Minimum night temperature ranging between } \\
12.5^{\circ} \mathrm{C}-23^{\circ} \mathrm{C}\end{array}$} & \multicolumn{6}{|c|}{$\begin{array}{l}\text { Minimum night temperature ranging between } \\
2^{\circ} \mathrm{C}-7.5^{\circ} \mathrm{C}\end{array}$} \\
\hline & $\mathrm{NF} / \mathrm{C}$ & $\begin{array}{l}\text { FW } \\
\text { (g) }\end{array}$ & $\begin{array}{l}\text { FD } \\
(\mathrm{mm})\end{array}$ & $\mathrm{NS} / \mathrm{F}$ & $\begin{array}{l}\mathrm{Y} / \mathrm{C} \\
(\mathrm{kg})\end{array}$ & $\begin{array}{l}\mathrm{Y} / \mathrm{P} \\
(\mathrm{kg})\end{array}$ & $\mathrm{NF} / \mathrm{C}$ & $\begin{array}{l}\text { FW } \\
(\mathrm{g})\end{array}$ & $\begin{array}{l}\text { FD } \\
(\mathrm{mm})\end{array}$ & $\mathrm{NS} / \mathrm{F}$ & $\begin{array}{l}\mathrm{Y} / \mathrm{C} \\
(\mathrm{kg})\end{array}$ & $\begin{array}{l}\mathrm{Y} / \mathrm{P} \\
(\mathrm{kg})\end{array}$ \\
\hline V1T1 (Sahel, 0ppm) & $3.20 f$ & $112.40 \mathrm{i}$ & $52.95 \mathrm{~h}$ & $58.90 \mathrm{a}$ & $0.37 \mathrm{~g}$ & $5.92 \mathrm{~g}$ & $2.00 \mathrm{~g}$ & $51.37 \mathrm{i}$ & $43.46 \mathrm{~h}$ & $54.40 \mathrm{a}$ & $0.11 \mathrm{j}$ & $2.35 \mathrm{j}$ \\
\hline V1T2 (Sahel, 25 ppm single spray) & $4.20 \mathrm{e}$ & $131.22 \mathrm{fg}$ & $61.00 \mathrm{f}$ & $42.80 \mathrm{~b}$ & $0.52 \mathrm{f}$ & $6.36 f$ & $3.40 \mathrm{f}$ & $65.36 \mathrm{~h}$ & $48.62 \mathrm{~g}$ & $21.60 \mathrm{ef}$ & $0.22 \mathrm{~h}$ & $2.72 \mathrm{~h}$ \\
\hline V1T3 (Sahel, 50 ppm single spray) & $5.00 \mathrm{~d}$ & $139.74 \mathrm{ef}$ & $63.11 \mathrm{~d}$ & $32.40 \mathrm{c}$ & $0.68 \mathrm{e}$ & $8.16 \mathrm{e}$ & $4.40 \mathrm{e}$ & $78.48 \mathrm{de}$ & $51.02 \mathrm{f}$ & $18.80 \mathrm{fg}$ & $0.34 \mathrm{ef}$ & $4.14 \mathrm{ef}$ \\
\hline V1T4 (Sahel, 75 ppm single spray) & $5.60 c$ & $157.84 \mathrm{~cd}$ & $68.86 \mathrm{ab}$ & $26.00 \mathrm{de}$ & $0.88 b$ & $10.65 b$ & $5.20 \mathrm{bc}$ & $88.58 b c$ & $54.90 \mathrm{cde}$ & $7.40 \mathrm{i}$ & $0.47 \mathrm{c}$ & $5.67 \mathrm{c}$ \\
\hline V1T5 (Sahel, 25 ppm double spray) & $4.20 \mathrm{e}$ & $159.35 c$ & $65.57 \mathrm{c}$ & $32.80 \mathrm{c}$ & $0.68 \mathrm{e}$ & $8.18 \mathrm{e}$ & $4.40 \mathrm{e}$ & $74.15 f$ & $51.94 f$ & 16.20gh & $0.31 \mathrm{fg}$ & $3.82 \mathrm{fg}$ \\
\hline V1T6 (Sahel, 50 ppm double spray) & $4.80 \mathrm{~d}$ & $175.33 b$ & $67.58 b$ & $24.40 \mathrm{de}$ & $0.81 b c$ & $9.82 \mathrm{bc}$ & $5.00 \mathrm{~cd}$ & $86.19 \mathrm{c}$ & $52.99 \mathrm{def}$ & $13.40 \mathrm{~h}$ & $0.44 \mathrm{~cd}$ & $5.38 \mathrm{~cd}$ \\
\hline V1T7 (Sahel, 75 ppm double spray) & $5.80 \mathrm{bc}$ & $187.37 \mathrm{a}$ & $69.32 \mathrm{a}$ & $18.80 f$ & $1.11 \mathrm{a}$ & $13.35 \mathrm{a}$ & $5.70 \mathrm{a}$ & $96.79 \mathrm{a}$ & $59.79 \mathrm{~b}$ & $3.00 \mathrm{j}$ & $0.58 \mathrm{a}$ & $7.05 \mathrm{a}$ \\
\hline V2T1 (Saandal, 0ppm) & $4.20 \mathrm{e}$ & 114.18hi & $51.81 \mathrm{~h}$ & $55.20 \mathrm{a}$ & $0.48 \mathrm{fg}$ & $7.07 \mathrm{fg}$ & $3.00 \mathrm{f}$ & $65.22 \mathrm{~h}$ & $47.95 \mathrm{~g}$ & $43.80 \mathrm{~b}$ & $0.18 \mathrm{i}$ & 3.191 \\
\hline V2T2 (Saandal, 25 ppm single spray) & $4.80 \mathrm{~d}$ & 119.58hi & $57.39 \mathrm{~g}$ & $43.40 \mathrm{~b}$ & $0.55 f$ & $7.71 \mathrm{f}$ & $4.20 \mathrm{e}$ & $69.87 \mathrm{~g}$ & $51.86 f$ & $34.400 \mathrm{c}$ & $0.30 \mathrm{~g}$ & $3.63 \mathrm{~g}$ \\
\hline V2T3 (Saandal, 50 ppm single spray) & $5.60 \mathrm{c}$ & $124.94 \mathrm{gh}$ & $60.07 f$ & $34.80 \mathrm{c}$ & $0.69 \mathrm{de}$ & $8.28 \mathrm{de}$ & $5.00 \mathrm{~cd}$ & $77.01 \mathrm{ef}$ & $55.06 \mathrm{~cd}$ & $26.00 \mathrm{~d}$ & $0.37 \mathrm{e}$ & $4.55 \mathrm{e}$ \\
\hline V2T4 (Saandal, 75 ppm single spray) & $6.20 \mathrm{~b}$ & $136.19 f$ & $62.63 \mathrm{de}$ & $26.40 \mathrm{de}$ & $0.86 \mathrm{~b}$ & $10.37 \mathrm{~b}$ & $5.60 \mathrm{ab}$ & $91.36 \mathrm{~b}$ & $59.22 \mathrm{~b}$ & $25.00 \mathrm{de}$ & $0.51 \mathrm{~b}$ & $6.14 \mathrm{~b}$ \\
\hline V2T5 (Saandal, $25 \mathrm{ppm}$ double spray) & $5.60 \mathrm{c}$ & $131.18 \mathrm{fg}$ & $61.29 \mathrm{ef}$ & $24.60 \mathrm{de}$ & $0.72 \mathrm{cde}$ & $8.75 \mathrm{cde}$ & $4.60 \mathrm{de}$ & $75.28 \mathrm{ef}$ & $52.88 \mathrm{ef}$ & 24.60de & $0.36 \mathrm{e}$ & $4.33 \mathrm{e}$ \\
\hline V2T6 (Saandal, 50 ppm double spray) & $6.00 \mathrm{bc}$ & $134.21 \mathrm{fg}$ & $62.92 \mathrm{~d}$ & $27.20 \mathrm{~d}$ & $0.79 \mathrm{bcd}$ & $9.57 \mathrm{bcd}$ & $5.60 \mathrm{ab}$ & $80.42 d$ & $55.66 \mathrm{c}$ & $24.40 \mathrm{de}$ & $0.43 \mathrm{~d}$ & $5.24 \mathrm{~d}$ \\
\hline V2T7 (Saandal, 75 ppm double spray) & $7.80 \mathrm{a}$ & $148.31 \mathrm{de}$ & $65.60 \mathrm{c}$ & $22.60 \mathrm{ef}$ & $1.13 \mathrm{a}$ & $13.65 \mathrm{a}$ & $6.30 \mathrm{a}$ & $95.55 \mathrm{a}$ & $62.74 \mathrm{a}$ & $16.00 \mathrm{gh}$ & $0.59 \mathrm{a}$ & $7.11 \mathrm{a}$ \\
\hline LSD & 0.56 & 10.81 & 1.57 & 4.56 & 0.11 & 1.32 & 0.48 & 3.22 & 2.14 & 1.34 & 2.10 & 0.41 \\
\hline Level of significance (5\%) & * & * & $*$ & ns & ns & ns & ns & * & ns & * & * & * \\
\hline
\end{tabular}

NF/C: Number of fruit per cluster; FW: Fruit weight; FD: Fruit Diameter; NS/F: No. of seed fruit; Y/C: Yield per cluster; Y/P: yield per plant.

When minimum night temperature was $12.5^{\circ} \mathrm{C}-23^{\circ} \mathrm{C}$ then the double spray of $75 \mathrm{ppm}$ caused more gain in yield per cluster $(1.12 \mathrm{~kg})$ than other treatments especially control (0.42). Double spray treatment of $25 \mathrm{ppm}(0.70 \mathrm{~kg})$ and $50 \mathrm{ppm}(0.80 \mathrm{~kg})$ showed more yield than the single spray treatment of $25 \mathrm{ppm}(0.54$ $\mathrm{kg})$ and $50 \mathrm{ppm}(0.68 \mathrm{~kg})$. Single spray of $75 \mathrm{ppm}$ $(0.87 \mathrm{~kg})$ was found more effective than other single spray treatments to increase yield per cluster i.e. 25 ppm $(0.54 \mathrm{~kg})$, and $50 \mathrm{ppm}(0.68 \mathrm{~kg})$. It was noted that in the sprayed cluster mostly fruits were formed by bypassing the regular fruit development process in which pollination and fertilization are the main requirements for fruit set. An increase in yield by the application of growth regulators under the cool season was also observed by Abad and Guardiola (1985). Gemici et al. (2006) and Ramin (2003) reported that parthenocarpic fruits development, increase in fruit weight and fruit size (fruit diameter) were the main reasons for more yield of plants that were treated with 4-CPA.

\section{Number of fruits per cluster}

The number of fruits per cluster is an important parameter that contributes to yield. As more the number of fruit sets per cluster more will be the yield of the plant. During cool nights when minimum night temperature was $2^{\circ} \mathrm{C}-7.5^{\circ} \mathrm{C}$, the interaction effects of variety into 4-CPA levels were non-significant indicating that the main effects of variety and 4-CPA levels are more important in respect of the number of fruit per cluster. Double sprayed treatments set more number of fruits per cluster than single spray when minimum night temperature was between $2^{\circ} \mathrm{C}-7.5^{\circ} \mathrm{C}$. More number of fruits (6.00) was set by applying double spray of $75 \mathrm{ppm}$ (T7) while fewer fruits per cluster (2.5) were set in control clusters on which no chemical dose was sprayed (Table 3). In single spray treatment, by applying $75 \mathrm{ppm}$ concentration more number of fruits (5.90) were sets as compared to other treatments i.e $25 \mathrm{ppm}$ (3.80), and $50 \mathrm{ppm}$ (4.70) when crop faced the temperature of $2^{\circ} \mathrm{C}-7.5^{\circ} \mathrm{C}$ in the night (Table 3). Saandal variety was able to set $10.5 \%$ more fruits (4.85) per cluster as compared to Sahel (4.34) when night temperature was ranged between $2^{\circ} \mathrm{C}-7.5^{\circ} \mathrm{C}$ (Table 2).

When minimum night temperature was $12.5^{\circ} \mathrm{C}-23^{\circ} \mathrm{C}$, double spray treatment of $75 \mathrm{ppm}(6.80)$ showed more number of fruit per clusters than other double spray treatments i.e. $25 \mathrm{ppm}$ (4.90) and $50 \mathrm{ppm}$ (5.40). However, these double sprayed treatments did not show significant differences in comparison with single spray treatment $25 \mathrm{ppm}$ (4.50) and $50 \mathrm{ppm}$ (5.30). In 
single sprays, 75 ppm was found more effective than other treatments to increase the number of fruits per cluster i.e. 25 ppm (4.50), and 50 ppm (5.30). More number of fruits in treated clusters were mainly due to parthenocarpy that bypassed the process of natural pollination and fertilization which are two major processes required for proper fruit setting through sexual processes.

\section{Fruit weight}

In cooly nights when the temperature was $2{ }^{\circ} \mathrm{C}$ $-7.5^{\circ} \mathrm{C}$ at night time, Sahel hybrid $(51.37 \mathrm{~g})$ shows more reduction in fruit weight than Saandal (65.22) as compared to nights when the temperature was between $12.5^{\circ} \mathrm{C}-23^{\circ} \mathrm{C}$ in which Sahel fruit weight was $112.40 \mathrm{~g}$ and Saandal was $114.18 \mathrm{~g}$. An increase in fruit weight by the application of different doses of 4-CPA was observed in both temperature conditions. During cool nights when minimum night temperature was $2^{\circ} \mathrm{C}-7.5^{\circ} \mathrm{C}$, interaction effects of variety into $4-\mathrm{CPA}$ levels were significant indicating that the response of different 4-CPA levels was highly dependent on varieties. With a double spray of 75 ppm, Sahel hybrid (96.79) depicted more gain in fruit weight than Saandal (95.55). Overall 46.9\% increase in Sahel (96.79 g) and $31.74 \%$ in Saandal fruit weight $(95.55 \mathrm{~g})$ occurred with a double spray of 75 ppm (Table 4). Mean comparison of single and double sprayed treatments regarding fruit weight showed that double sprayed treatments were more effective in increasing fruit weight than single spray treatments in both varieties. An experiment performed by Gemici et al. (2006) also showed congruent results with our studies and reported an increase in fresh as well as dry fruit weight of the plants that were treated with 4-CPA.

\section{Fruit diameter}

An increase in fruit diameter by the application of 4-CPA over control in both temperature conditions was recorded. Highest fruit diameter $(67.46 \mathrm{~mm}, 61.26$ $\mathrm{mm}$ ) was observed by the use of 75 ppm concentration two times in a week in both temperature conditions $12.5^{\circ} \mathrm{C}-23^{\circ} \mathrm{C}$ and $2^{\circ} \mathrm{C}-7.5^{\circ} \mathrm{C}$, respectively. Similarly, minimum fruit diameter $(52.38 \mathrm{~mm}, 45.71 \mathrm{~mm})$ was found in control $(0 \mathrm{ppm})$ in both temperature conditions $12.5^{\circ} \mathrm{C}-23^{\circ} \mathrm{C}$ and $2^{\circ} \mathrm{C}-7.5^{\circ} \mathrm{C}$, respectively (Table 3). Both varieties showed different responses towards different 4-CPA levels but maximum fruit diameter was recorded in both varieties when treated with $75 \mathrm{ppm}$ twice in a week (Table 2). The results of this study are in agreement with the findings of Halder et al. (2003) who reported the application of 4-CPA increased the fruit length as well as fruit diameter of summer tomato. Gemici et al. (2006) also observed an increase in fruit diameter by the application of 4-CPA and on the basis of hormonal assay he linked this increase with the increase in the internal hormone Indol-3-acetic acid IAA of the sprayed plants.

\section{Number of seed per fruit}

Parthenocarpic fruit development is the main reason for increase fruit set per cluster and yield. The decrease in seed per fruit was observed with the application of growth regulators. Less seed formation in sprayed fruits indicates the effectiveness of hormone in parthenocarpic fruit development. The highest number of seeds $(57.05,39.50)$ was observed in fruits of the control cluster in which no spray occurred and the minimum number of seeds $(20.70,9.50)$ was found in the fruits that were treated with $75 \mathrm{ppm}$ concentration twice in a week (Table 3). Karapons et al. (2008) reported the parthenocarpic fruit development in the fruit clusters that were treated with 4-CPA. Gemici et al. (2006) found $80 \%$ parthenocarpic fruits in the plants that were treated with 4-CPA. Moreover, they reported that these seedless fruits were formed in control as well as treated clusters as ovaries of these flowers did not wait for pollination and form fruit bypassing the process of pollination and fertilization.

As in the case of hormonal application, the ovary has not waited for signal receipt on account of natural fertilization to develop into fruit and fewer seeds in these fruits may be due to delayed or disturbance in pollen tube growth on account of ovary enlargement. Whereas in case of control treatment, stigma as well as pollen tube development has remained active for the large time, thus allowing natural fertilization even after prolonged time and resulted in more seed formation as compared to sprayed one.

\section{Conclusions and Recommendations}

Based on this study it may be concluded that increase in yield of winter tomato planting is possible during low temperature stress when minimum night temperature was ranging between $12.5^{\circ} \mathrm{C}-23^{\circ} \mathrm{C}$ and $2^{\circ} \mathrm{C}-7.5^{\circ} \mathrm{C}$, by the use of growth regulator 4-CPA at $75 \mathrm{ppm}$ two times in a week. A positive influence 
of 4-CPA describes above on tomato yield could be a tool for getting economical tomato yield in the low night temperature stress conditions.

\section{Authors Contributions}

Saeed Ahmed Shah Chishti, Saba Aleem, executed the whole experiment, collected and analyzed the data and also wrote the whole manuscript. Kashif Nadeem helped in data collection. Nusrat Parveen and Iram Sharif helped in data, analyzing and write-up of the manuscript. Muhammad Najeebullah monitored the whole experiment and provided technical help in performing the experiment

\section{Conflict of interest}

The author(s) declare(s) that there is no conflict of interest regarding the publication of this article.

\section{References}

Abad, M. and J.L. Guardiola. 1985. Fruit-set and development in the tomato (Lycopersicon esculentum Mill.) grown under protected conditions during the cool season in the southeastern coastal region of Spain. The response to exogenous growth regulators. In a symposium on protected cultivation of solanacea in mild winter climates, Albufeira, Portugal. 191: 123-132. https://doi.org/10.17660/ ActaHortic.1986.191.12

Adams, S.R., K.E. Cockshull and C.R.J. Cave. 2001. Effect of temperature on the growth and development of tomato fruits. Ann. Bot. 88(5): 869-877. https://doi.org/10.1006/ anbo.2001.1524

Fernandez-Munoz, R., J.J.G. Fernandez and J. Cuartero. 1995. Variability of pollen tolerance to low temperatures in tomato and related wild species. J. Hortic. Sci. 70(1): 41-49. https://doi. org/10.1080/14620316.1995.11515271

Gemici, M., T. Bengu and T. Kit. 2006. Effects of 2, 4-D and 4-CPA on yield and quality of the tomato, Lycopersicon esculentum Miller. J. Fac. Sci. 29: 24-32.

Halder, N.K., M.K. Shaha, A.F. Kabir, J.U. Sarker and M.J. Rahman. 2003. Response of summer tomato to hormone and planting time at hill slope. Pak. J. Biol. Sci. 6: 146-148. https://doi. org/10.3923/pjbs.2003.146.148

Karapanos, I.C., S. Mahmood and C. Thanopoulos. 2008. Fruit set in solanaceous vegetable crops as affected by floral and environmental factors. The Eur. J. Plant Sci. Biotechnol. 2(1): 88-105.

Picken, A.J.F. and M. Grimmett. 1986. The effects of two fruit setting agents on the yield and quality of tomato fruit in glasshouses in Winter. J. Hortic. Sci., 61(2): 243-250. https://doi.org/1 0.1080/14620316.1986.11515697

Pramanik, K. and P.P. Mohapatra. 2017. Role of auxin on growth, yield and quality of tomato. A Review. Int. J. Curr. Microbiol. Appl. Sci. 6(11): 1624-1636. https://doi.org/10.20546/ ijcmas.2017.611.195

Pressman,E., R. Shaked and N. Firon.2007. Tomato (Lycopersicon esculentum) response to heat stress: focus on pollen grains. Plant Stress, 1: 216-227.

Rahman, M., M.A. Nahar, M.S. Sahariar and M.R. Karim. 2015. Plant growth regulators promote growth and yield of summer tomato (Lycopersicone sculentum Mill.), Progress. Agric 26: 32-37.

Ramin, A., 2003. Effects of auxin application on fruit formation in tomato growing under stress temperatures in the field. J. Hortic. Sci. Biotechnol. 78(5): 706-710. https://doi.org/10 1080/14620316.2003.11511687

Tiwari, A.K. and D.K. Singh. 2014. Use of plant growth regulators in tomato (Solanum lycopersicum L.) under tarai conditions of Uttarkhand. Indian J. Hill Farming, 27(2): 3840. 